\title{
Raoultella terrigena
}

National Cancer Institute

\section{Source}

National Cancer Institute. Raoultella terrigena. NCI Thesaurus. Code C86717.

A species of Gram negative, rod shaped bacteria assigned to the phylum Proteobacteria.

This species is ornithine decarboxylase negative, cannot deaminate tryptophan, is able to grow at 10 degrees Celsius and uses sorbose as a carbon source. R. terrigena is a rare pathogen. 